\title{
Distribuição espacial e temporal de Cetengraulis edentulus (Cuvier) (Actinopterygii, Engraulidae) na Baía de Sepetiba, Rio de Janeiro, Brasil
}

\author{
Márcio de Araújo Silva, Francisco G. Araújo, Márcia C. Costa de Azevedo \& Pablo Mendonça
}

Laboratório de Ecologia de Peixes, Posto de Aqüicultura, Universidade Federal Rural do Rio de Janeiro. Antiga Rodovia RioSão Paulo, Km 47, 23851970 Seropédica, Rio de Janeiro, Brasil. E-mail: marciobio@ig.com.br; gerson@ufrrj.br

\begin{abstract}
Spatial and temporal distribution of Cetengraulis edentulus (Cuvier) (Actinopterygii-Engraulidae) in the Sepetiba Bay, Rio de Janeiro, Brazil. Anchovies are members of the Engraulidae family, characterized to present coastal pelagic habits, concentrating in large schoolings distributed among the continental shelf and semi-closed environment, like bays, where they are target of heavy fisheries. The present study aims to describe spatial and temporal distribution of Cetengraulis edentulous (Cuvier, 1828) in the Sepetiba bay $\left(22^{\circ} 54^{\prime}-23^{\circ} 04^{\prime}{ }^{\prime}\right.$, $43^{\circ} 34^{\prime}-44^{\circ} 10^{\prime} \mathrm{W}$ ) and to assess influences of environmental variables on fish occurrence. A monthly sampling programme was carried out between October 1998 and September 1999, to take both, fish and environmental information on temperature, salinity and depth. Three bay zones were established based on spatial gradient of salinity and depth. Adults $C$. edentulus were more abundant in the inner bay zone; seasonally, larger size groups (total length $>16 \mathrm{~cm}$ ) occurred in spring/summer. Significant negative correlations were found between fish abundance and salinity and depth. The close relationship of this species to innermost areas in bays, and the absence of juveniles in sandy beaches, suggest that young-of-the-year use mangrove areas as recruitment sites.
\end{abstract}

KEY WORDS. Anchovies, coastal fishes, distritubion, Engraulidae, Sepetiba bay.

Os peixes da família Engraulidae, vulgarmente conhecidos como manjubas e sardinhas boca-torta, apresentam ampla distribuição geográfica, sendo encontrados predominantemente em águas tropicais e sub-tropicais das Américas (McGowAN \& BERRY 1983). São abundantes em regiões costeiras semi-abertas, como baías, que funcionam como áreas de criação nas primeiras fases de vida. A desova em geral ocorre nas zonas costeiras da plataforma com os ovos e formas larvais sendo transportados para baías onde encontram melhores condições de proteção e disponibilidade de alimento (Сото et al. 1988, MACGREGOR \& Houde 1996), apresentam ciclo de vida relativamente curto e, quando adultos, realizam movimentos de curta extensão entre a plataforma interna adjacente e o interior de baías. Desempenham importante papel na cadeia alimentar dos oceanos, servindo de forragem a muitas espécies de peixes e aves marinhas (HILDEBRAND 1963), e sendo um dos principais contribuintes do fluxo de energia ao longo da cadeia alimentar, visto que são os maiores consumidores de zooplancton entre os peixes (BAird \& Ulanowicz 1989).

As pescarias de Engraulidae na plataforma continental assumem grande importância em termos de produção. O Peru destaca-se por apresentar uma das maiores pescarias do mundo, baseada principalmente na produção de Engraulis ringens Jenyns, 1842. Em 1972 foram capturados 12 milhões de toneladas desta espécie, equivalente a $22 \%$ das capturas mundiais
(VALENTIN 1994). Cita-se que outras espécies do gênero Engraulis Cuvier, 1816 também apresentam grande importância comercial, como Engraulis anchoita Hubbs \& Marini, 1935 na costa do Uruguai e da Argentina (Sanchez \& De-Ciechomski 1995), Engraulis mordax Girard, 1854 no norte e nordeste do México e Cetengraulis edentulus (Cuvier, 1828) no leste da Venezuela (Сото et al. 1988). Fischer (1978 apud Silva \& Araújo 2000) relatou uma captura total de $1226 \mathrm{t}$ de C. edentulus em 1975 em toda área de pesca 31 da FAO (Atlântico - centro oeste). No Brasil, pescarias de Anchoviella Fowler, 1911 são comuns em estuários e trechos baixos dos rios Ribeira de Iguape e no estuário de São Vicente, ambientes utilizados por este gênero para desova (Bendazoli et al. 1990, Paiva-Filho et al. 1986, 1990). No Rio Paraíba do Sul também é realizada uma pesca intensiva de Anchoviella lepidentostole (Fowler, 1911) no trecho baixo, próximo à localidade a São Fidelis (Araújo 1996). Na Baía de Sepetiba a pesca da manjuba é feita basicamente sobre Anchoa tricolor (Agassiz, 1829) e C. edentulus.

O ciclo de vida de $A$. tricolor foi descrito por Silva \& ARAújo (2000), porém pouco se sabe sobre $C$. edentulus, que vem sofrendo intensiva pesca nos últimos anos devido ao incentivo do Governo Federal para captura de exemplares que são industrializados e comercializados no Nordeste do Brasil, visando suprir as deficiências da pesca da sardinha. Diferentemente das espécies do gênero Anchoa Jordan \& Evermann, 1927, 
jovens do ano de C. edentulus não recrutam nas praias arenosas da Baía de Sepetiba, conforme registros da década de 90; nos anos 80 apenas registros ocasionais foram feitos para esta espécie na área (SERGIPENSE \& SAZIMA 1995).

A Baía de Sepetiba tem sido reportada como importante área de recrutamento e desenvolvimento dos Engraulidae, especialmente nas praias arenosas da zona de maior influência do mar (ARAújo et al. 1997, 1998, Pessanha et al. 2000). Mecanismos de coexistência de populações visando evitar a exclusão competitiva são basicamente a separação trófica, espacial ou temporal (Ross 1986). No caso de peixes caracteristicamente planctônicos como os Engraulideos, grupos que não se segregam espacialmente, apresentam diferentes preferências alimentares. SERGIPENSE et al. (1999) encontraram que Anchoa januaria (Steindachner, 1879) e C. edentulus, que são comuns nas áreas mais internas da Baía de Sepetiba, apresentam formas distintas de obtenção do alimento, com a primeira espécie sendo predominantemente zooplanctófaga e a segunda, fitoplanctófaga. Uma segregação espacial entre juvenis de $A$. januaria e $A$. tricolor, espécies dominantes nas praias, foi detectada por SiLVA \& AraúJo (2000). Diferenças na abundância relativa das espécies de Engraulidae tem sido encontrado nos diferentes tipos de amostragens (arrasto de praia, arrasto de fundo e cerco de meia água) por Araújo et al. 2002. C. edentulus é reportada como único representante de Engraulidae a apresentar contribuição relevante nos arrastos de fundo, estando praticamente ausente nos cercos de meia água praticados na zona externa da Baía ou nos arrastos de praia, realizados ao longo de toda a margem continental da Baía. O presente trabalho visa determinar a distribuição espacial e temporal de C. edentulus na Baía de Sepetiba com base nos arrastos de fundo, além de verificar as possíveis influências das variáveis ambientais na sua distribuição.

\section{MATERIAL E MÉTODOS}

\section{Área de estudos}

A Baía de Sepetiba (Fig. 1) apresenta forma alongada, limitando-se a norte e a leste pelo continente, ao sul pela Restinga de Marambaia e a oeste pela Baía de Ilha Grande. A bacia hidrográfica ocupa uma área de cerca de $2500 \mathrm{~km}^{2}$, e o espelho d'água apresenta uma área de $520 \mathrm{~km}^{2}$. A baía apresenta sedimentos de origem fluvial, de canais de maré e de manguezal. Em termos geológicos ela é caracterizada como bacia semiconfinada, tendendo ao fechamento (Barroso 1989). Verifica-se a presença de duas áreas distintas na baía, uma mais interna, que sofre grande influência dos rios e canais de marés, outra externa, com maior influência das águas marinhas. $\mathrm{O}$ clima enquadra-se no grupo A da Classificação de Köeppen, definido como Clima Tropical, diferenciando-se em função do contraste de topografia entre a área plana da baixada e as encostas que a envolvem. O tipo Aw caracterizado por verão úmido e inverno seco dominam a área plana da baixada. As maiores intensidades de chuvas são em dezembro-janeiro, alongando-se por vezes até março. O período seco é de maio a setembro (BARbiére \& KRONEMBERger 1994).

\section{Programa de amostragem}

Foram estabelecidas três zonas na Baía em função de um gradiente de profundidade e salinidade: 1) Zona interna, com profundidade inferior a $5 \mathrm{~m}$, localizando-se na região mais in-

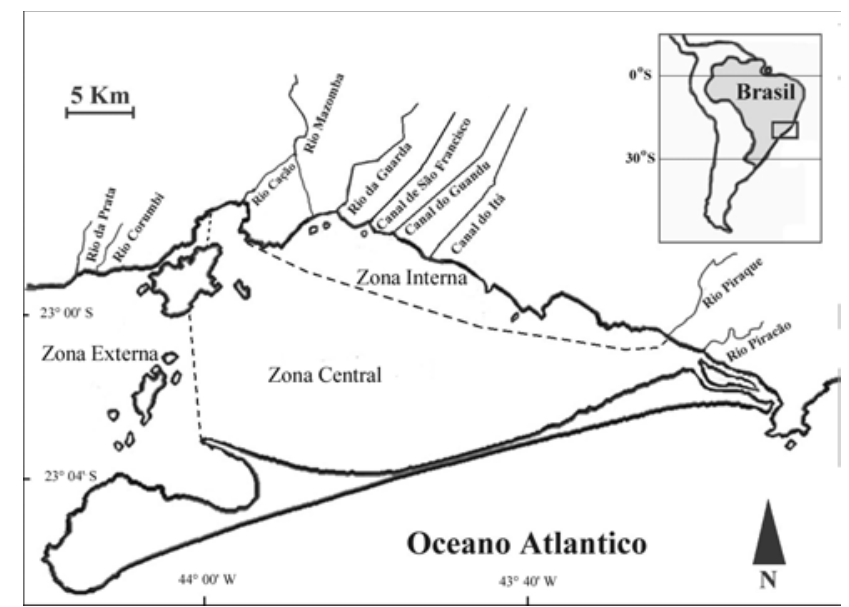

Figura 1. Área de estudo, Baía de Sepetiba, Rio de Janeiro, com indicações das zonas interna, central, externa, e dos rios que deságuam na baía. Escala 1: 260.000.

terna da Baía, com influência de aporte de rios e canais de maré; 2) Zona central, com profundidade entre 5 e $10 \mathrm{~m} \mathrm{e}$; 3) Zona externa, com profundidade maior que $10 \mathrm{~m}$, localizando-se na área mais externa da Baía, com maior influência de águas oceânicas. Foram, selecionados três locais aleatoriamente em cada zona, onde foram utilizados mensalmente nos arrastos de fundo com auxílio de barco com $12 \mathrm{~m}$ de comprimento, provido com rede de arrasto de portas com: tralha superior $=10,5 \mathrm{~m}$; tralha inferior $=12,0 \mathrm{~m}$; e nas asas malha de $25 \mathrm{~mm}$ entre nós e de $15 \mathrm{~mm}$ no ensacador. As amostragens foram realizadas no período diurno, com duração de 20 minutos, a uma velocidade de dois nós, cobrindo uma extensão de aproximadamente $1,5 \mathrm{Km}$. Em todas as amostras foram aferidas a temperatura e a salinidade de fundo e a profundidade.

\section{Análise de dados}

A captura por unidade de esforço-CPUE (número de peixes/arrasto) foi utilizada como indicador da abundância relativa. Comparações espaciais e sazonais (outono: março/maio; inverno: junho/agosto; primavera: setembro/novembro; e verão dezembro/fevereiro) foram feitos para as CPUEs e para as variáveis ambientais, utilizando a ANOVA Bi-fatorial Modelo 2, com os dados brutos tendo sido previamente logaritimizados $\left(\log _{10}\right.$ $X+1$ ) para atender os requisitos de ANOVA. CPUEs por intervalos de classe de temperatura da água, de salinidade e profundidade também foram comparadas (Tab. I). O teste de diferenças de médias de Student-Newman-Keuls (SNK) foi utilizado sempre que a Hipótese Nula foi rejeitada (SOKAL \& RoHLF 1981).

\section{RESULTADOS}

Composição da captura. Cetengraulis edentulus foi à espécie de Engraulidae mais capturada, com 1568 indivíduos amostrados nas 107 amostragens de arrasto de fundo, o que representou $88,63 \%$ desta família. Os indivíduos foram basicamente subadultos e adultos e apresentaram tamanhos variando entre 7 e $20 \mathrm{~cm}$ de comprimento total. 
Tabela I. Intervalos de classes para os parâmetros ambientais analisados na Baía de Sepetiba, Rio de Janeiro.

\begin{tabular}{cccc}
\hline Intervalos & Temperatura $\left({ }^{\circ} \mathrm{C}\right)$ & Salinidade & Profundidade $(\mathrm{cm})$ \\
\hline 1 & $<20,1$ & $<27,1$ & $<500$ \\
2 & $20,1-23,0$ & $27,1-30,0$ & $500,1-1000$ \\
3 & $23,1-26,0$ & $30,1-33,0$ & $1000,1-2000$ \\
4 & $>26,0$ & $>33,0$ & $>2000$ \\
\hline
\end{tabular}

Distribuição espacial. Diferenças significativas $(\mathrm{p}<0,05)$ foram apresentadas nas CPUEs com maiores valores na zona interna; nas zonas central e externa estes peixes foram registrados apenas ocasionalmente (Tab. II).

Tabela II. Diferenças significativas ( $p<0,05$ ONEWAY) da distribuição de Cetengraulis edentulus em função dos locais de coleta, variáveis ambientais da Baía de Sepetiba, Rio de Janeiro, através da análise de variâncias e teste de Student-Newman-Keuls (SNK).

\begin{tabular}{lccc}
\hline \multicolumn{1}{c}{ Variáveis } & Valores de $F$ & \multicolumn{2}{c}{ Diferenças } \\
\hline \multirow{2}{*}{ Locais de coleta } & $24,9^{* *}$ & Zona interna $>$ & Zona central e externa \\
& & $(43,0)$ & $(0,02-0,36)$ \\
Salinidade & $15,5^{* *}$ & $1>$ & $2,3,4$ \\
& & $(132,0)$ & $(0,04-23,4)$ \\
\hline
\end{tabular}

Distribuição sazonal. Não foram detectadas diferenças sazonais significativas nas CPUEs quando consideradas todas as amostras em conjunto. Um forte gradiente temporal, no entanto foi possível de ser observado na estrutura de tamanho ao longo dos meses amostrados, com indivíduos de maior tamanho $(\mathrm{CT}>16 \mathrm{~cm}$ ) ocorrendo em grande abundância entre outubro e janeiro; nos demais meses as abundâncias relativas foram bem menores e os indivíduos apresentaram menor tamanho $(\mathrm{CT}<16 \mathrm{~cm})$ (Fig. 2).

Influência das Variáveis Ambientais. Diferenças significativas nas CPUEs em relação à salinidade foram registradas, com maiores valores nas menores faixas (Tab. II). Para a temperatura e a profundidade não foram verificadas diferenças nas CPUEs por intervalo de classe destes parâmetros. Significativa correlação foi detectada entre as CPUEs e a profundidade e a salinidade, de acordo com r-Spearman; não foi observada correlação significativa entre as CPUEs e a temperatura (Tab. III).

\section{DISCUSSÃO}

Cetengraulis edentulus é uma espécie que utiliza a coluna d'água, mais especificamente aquela mais próxima ao fundo, concentrando-se em elevadas abundâncias na parte mais interna da Baía de Sepetiba, a qual é caracterizada por menores profundidades e salinidades. A presença desta espécie quase que exclusivamente nesta área mais interior da Baía, coincide

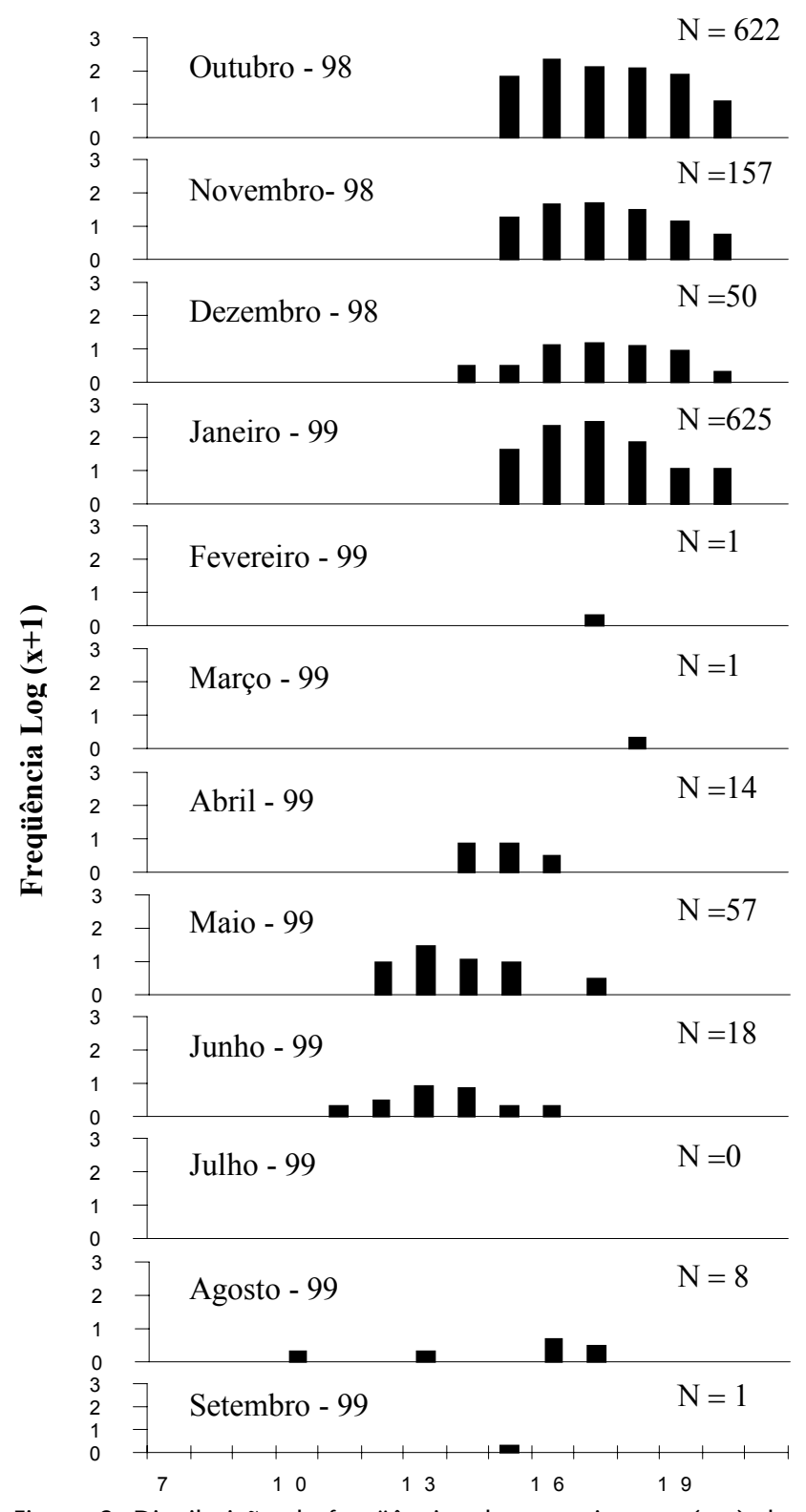

Figura 2. Distribuição de freqüências de comprimento $(\mathrm{cm})$ de Cetengraulis edentulus na zona interna da Baía de Sepetiba, Rio de Janeiro.

Tabela III. Correlação (r-Spearman) entre a abundância de Cetengraulis edentulus com a temperatura, salinidade e profundidade.

\begin{tabular}{lc}
\hline \multicolumn{1}{c}{ Variáveis } & Correlação (r-Spearman) \\
\hline Temperatura $\left({ }^{\circ} \mathrm{C}\right)$ & 0,112 \\
Salinidade & $-0,386^{* *}$ \\
Profundidade $(\mathrm{cm})$ & $-0,586^{* *}$ \\
\hline
\end{tabular}


com o indicado por Figueiredo \& Menezes (1978) que apontam a ocorrência destes peixes em áreas de baixa salinidade e até mesmo em ambientes dulcícolas. Diferentemente da maioria das espécies de Engraulidae, especialmente do gênero dominante Anchoa, esta espécie não usa as praias arenosas da zona externa da Baía como áreas de recrutamento (SiLVA \& ARAújo 2000).

O ciclo de vida de $C$. edentulus, especialmente os locais de reprodução e de recrutamento, é desconhecido. MAZETTI (1983 apud SERgIPENSE \& SAZIMA 1995) comenta que apesar da presença de pequena quantidade de formas juvenis dessa espécie na Baía de Guanabara, Rio de Janeiro, a presença de ovos é um indício de que todo seu ciclo vital ocorra nesta área. O autor também destacou a ocorrência de C. edentulus na Barra de Guaratiba, Rio de Janeiro, próximo de áreas de manguezais, leva a supor que parte do ciclo de vida desta espécie ocorra nestas áreas. GAY et al. (2000) verificaram a presença de indivíduos adultos e pós-larvas, com ausência de ovos na laguna de Itaipu. No presente trabalho foi verificado uma grande abundância de adultos no interior da Baía, enquanto os juvenis foram raros, um padrão semelhante ao encontrado na Baía de Guanabara por Mazetti (1983 apud Sergipense \& Sazima 1995), sugerindo que os juvenis não utilizam as praias arenosas da Baía para o desenvolvimento inicial. Outras áreas como as lagunas e manguezais poderiam servir de recrutamento para estes peixes que é característico de fundo de Baías, onde adultos destas espécies são abundantes.

Adultos de C. edentulus são os representantes mais abundantes nos arrasto de fundo da zona mais interna da Baía de Sepetiba, uma região que apresenta extensas formações de manguezais em sua zona mais interna, uma indicação de que esta área provavelmente pode ser usada como local de recrutamento desta espécie, embora amostragens não tenham sido realizadas nesses locais, visando comprovar tal hipótese. GAY et al. (2000) mostrou que a distribuição desta espécie na laguna de Itaipu foi associada ao fundo tipicamente lodoso.

A separação espacial de outras espécies da família Engraulidae observada na Baía de Sepetiba, pode ser reflexo de adaptação às diferenças condições ambientais entre as zonas da Baía ou uma estratégia para evitar competição. Neste escopo, C. edentulus é a espécie mais eurióica, com adaptações às condições mais variáveis de fundo de Baía, onde o ambiente é mais estressante, tirando proveito da mais elevada produtividade desta área que recebe maior aporte de matéria orgânica, trazida pela drenagem continental, resultando em mais densas formações de fitoplancton. Sergipense \& Sazima (1995) relataram que C. edentulus se diferencia de Anchoa januaria, a segunda mais abundante espécie na zona interna da Baía de Sepetiba, por separação trófica, com A. januaria alimentando-se de zooplancton enquanto que $C$. edentulus utiliza o fitoplancton. No manguezal de Itacorubi, SC, é atribuída à ocorrência de fundo lodoso a elevada abundância desta espécie (Clezar et al. 1993). As poucas ocorrências de recrutas de C. edentulus na Baia de Sepetiba, foram registradas para praias lamosas da zona interna da Baía como Ponta Grossa e Pedra de Guaratiba, ainda na década de 80 (Sergipense \& SAZIma 1995). Apenas adultos de C. edentulus foram capturados nos arrastos de fundo, visto que esta espécie é comum nos fundos lamosos. Silva \& Araújo (2000) reportaram apenas registros ocasionais de C. edentulus em programas de amostragens de praia (margem continental) e cerco (zona central e externa da Baia, próxima das ilhas), quando comparada com A. tricolor e A. januaria, mostrando desta forma a segregação do habitat pelas espécies de manjubas na Baía. Sazonalmente, adultos formam densos grupos entre outubro e janeiro, na zona interna da Baía em profundidades inferiores a $5 \mathrm{~m}$, enquanto indivíduos de menores tamanhos $(\mathrm{CT}<16 \mathrm{~cm})$ em menor abundância ocorrem nos demais meses, o que nos leva a sugerir que o grupo de maior tamanho penetrou na Baía de Sepetiba por necessidades reprodutivas/ alimentares, já que indivíduos de menores tamanhos só irão aparecer na Baía entre os meses de abril e agosto. SERGIPENSE \& SAZIMA (1995) reportaram que C. edentulus ocorre predominantemente no período frio (abril a setembro), na laguna de Itaipu, o que não coincidiu com as variações sazonais observadas neste trabalho. Estas maiores ocorrências são provavelmente constituídas por indivíduos juvenis, haja vista que o período reprodutivo desta espécie parece ser primavera/verão. A sazonalidade dos grupos adultos verificados neste trabalho leva a supor que esta espécie é freqüentadora sazonal dos fundos lodosos de baías, que utiliza como áreas de alimentação. As áreas de reprodução e recrutamento são ainda desconhecidas, necessitando que se investiguem novos locais de prováveis usos, principalmente os manguezais de fundo de baía, que poderiam servir de recrutamento, já que exemplares desta espécie, diferentemente de outras espécies de Engraulidae, não utiliza praias arenosas como locais de recrutamento.

\section{REFERÊNCIAS BIBLIOGRÁFICAS}

ARaújo, F.G. 1996. Composição e estrutura da comunidade de peixes do médio e baixo Rio Paraíba do Sul, RJ. Revista Brasileira de Biologia, São Carlos, 56 (1): 111-126.

Araújo, F.G.; A.G. Cruz-Filho; M.C.C. Azevedo; A.C.A. Santos \& L.A.M. Fernandes. 1997. Estrutura da comunidade de peixes jovens da margem continental da Baía de Sepetiba, RJ. Acta Biológica Leolpoldensia, São Leopoldo, 19 (1): 61-83.

Araújo, F.G.; A.G. Cruz-Filho; M.C.C. Azevedo \& A.C.A. Santos. 1998. Estrutura da comunidade de peixes demersais da Baía de Sepetiba, RJ. Revista Brasileira de Biologia, São Carlos, 58 (3): 417-430.

Araújo, F.G.; M.C.C. Azevedo; M.A. Silva; A.L.M. Pessanha; I.D. Gomes \& A.G. Cruz-FilHo. 2002. Environmental influences on the demersal fish assemblages in the Sepetiba Bay, Brazil. Estuaries, Kansas, 25 (3): 441-450.

Baird, D. \& R.E. Ulanowicz. 1989. The seasonal dynamics of the Chesapeake Bay. Ecology Monograph, Kansas, 59: 329364.

Barbiére, E.B. \& D.M.P. Kronemberger. 1994. Climatologia do Litoral Sul-Sudeste do Estado do Rio de Janeiro. Caderno de Geociências, Rio de Janeiro, 12: 57-73.

Barroso, L.V. 1989. Diagnóstico Ambiental para a Pesca de Águas Interiores no Estado do Rio de Janeiro. Rio de Janeiro, MINTER/IBAMA-RJ/ACUMEP, 177p.

Bendazoli, A.; C.L.D.B. Rossi-Wongtschowski; M.T.D. Giamas \& L. Frosch. 1990. A Manjuba (Anchoviella lepidentostole) no Rio Ribeira de Iguape: biologia, comportamento e avaliação de estoque. São Paulo, IBAMA/IOUSP/IP-SA/ SEMA, 116p.

Clezar, L.; S.M. Hostim; G.C. Ribeiro \& B.S.D. Ledo. 1983. Abundance and distribution of Cetengraulis edentulus (Cuvier, 1828) (Clupeiformes-Engraulidae) of the Itacorubi Man- 
grove, SC, Brazil. Arquivos de Biologia e tecnologia, Curitiba, 36: 567-576.

Coto, C.F.; A.O. Luna; A.L. Calvo \& F.Z. García. 1988. Abundancia de algunas especies de Anchoas en la laguna de Términos (México), estimada a través de la captura de huevos. Annal del Institute de Ciência del Mar y Limnologia da Universidad Autónoma de México, México, 15 (1): 125-134.

GAY, D.; S. Sergipense \& C.F.D. Rocha. 2000. Ocorrência e distribuição de Cetengraulis edentulus (Curvier) (Teleostei, Engraulidae) na Laguna de Itaipu, Niterói, Rio de janeiro, Brasil. Revista Brasileira de Zoologia, Curitiba, 17 (2): 463 472.

Figueiredo, J.L. \& N.A. Menezes. 1978. Manual de peixes marinhos do sudeste do Brasil, II- Teleostei (1). São Paulo, Museu de Zoologia, Universidade de São Paulo, 110p.

Hildebrand, S.F. 1963. Family Engraulidae. In Fishes of the Western North Atlantic. Memoiries Sears Foundation for Marine Research, New Haven, 1 (3): 152-249.

MACGregor, J.M.; E.D. Houde. 1996. Onshore-Offshore pattern and variability in distribution and abundance of bay anchovy Anchoa mitchilli eggs and larvae in Cheasapeake Bay. Marine Ecology Progress Series, Nordbunte, 138: 15-25.

McGowAN, M.F. \& F.H. BerRY. 1983. Clupeiformes: Development and Relationships. In Ontogeny and Systematics of Fishes - Ahlstrom Symposium. American Society of Icthyologist and Herpetolisgt, Califórnia, 8: 108-126.

Paiva-Filho, A.M.; M.L. Zani Teixeira \& P.K. Kinara. 1986. Contribuição ao conhecimento da Biologia da manjuba, Anchoviella lepidentostole (FOWLER, 1911), no estuário de São Vicente, SP (Osteichthyes, Engraulidae). Boletim do Instituto Oceanográfico, São Paulo, 34: 71-77.

Paiva-Filho, A.M.; R. Giannini; F.B.R. Neto. 1990. A pesca da manjuba Anchoviella lepidentostole (Engraulidae) no estuário de São Vicente, SP. Relatório Interno do Instituto Oceanográfico, São Paulo, 29: 1-6.

Pessanha, A.L.M. \& F.G. Araújo; M.C.C. Azevedo \& I.D. Gomes. 2000. Variações temporais e espaciais na composição e estrutura da comunidade de peixes jovens da Baia de Sepetiba, Rio de Janeiro. Revista Brasileira de Zoologia, Curitiba, 17 (1): 251-261.

Ross, S.T. 1986. Resource partitioning in fish assemblages: a review of field studies. Copeia, Kansas, 1986: 352-388.

SANCHEZ, R.P. \& J.D. De-CieCHOMSKI. 1995. Spawning and nursery grounds of pelagic fish species in the sea-shelf off Argentina and adjacent areas. In: International Syposium on middle sized Pelagic fish held in las Palmas de Gran Canaria 59 (3-4): 455-478.

Sergipense, S. \& I. SAZima. 1995. Variações sazonais de ocorrência e tamanho em duas espécies de Engraulididae (Osteichthyes) na Baía de Sepetiba, Rio de Janeiro. Revista Brasileira de Biologia, Curitiba, 55 (3): 491-501

Sergipense, S.; E.P. Caramaschi \& I. Sazima. 1999. Morfologia e hábitos alimentares de duas espécies de Engraulididae (Teleostei, Clupeiformes) na Baía de Sepetiba, Rio de Janeiro. Revista Brasileira de Oceanografia, São Paulo, 47 (2): 173-188.

Silva, M.A. \& F.G. Araújo. 2000. Distribution and relative abundance of anchovies (Clupeiformes-Engraulididae) in the Sepetiba Bay, Rio de Janeiro, Brazil. Brazilian Archives of Biology and Technology, Paraná, 43 (4): 379-385.

SOKAL, R.R. \& F.J. RoHLF. 1981. Biometry: the principales and pratice of statistic in biological research. San Francisco, W.H. Freman, $2^{\text {nd }}$ ed., $776 \mathrm{p}$.

Valentin, J.L. 1994. Ressurgência: Fonte de vida dos oceanos. Ciência Hoje, Rio de Janeiro, 15 (102): 19-25.

Recebido em 05.XII.2002; aceito em 07.X.2003. 